九州大学学術情報リポジトリ

Kyushu University Institutional Repository

\title{
Optimum design consideration for interferometric spin wave logic operations
}

Nakashima, Yusuke

Kyushu University, Department of Electronics

Nagai, Keita

Kyushu University, Department of Electronics

Tanaka, Terumitsu

Kyushu University, Department of Electronics

Matsuyama, Kimihide

Kyushu University, Department of Electronics

http://hdl. hand le. net/2324/25565

出版情報: Journal of Applied Physics. 109 (7), pp.07D318(1)-07D318(3)，2011-03-25. American Institute of Physics

バージョン:

権利関係: (C) 2012 American Institute of Physics 


\title{
Optimum design consideration for interferometric spin wave logic operations
}

\author{
Y. Nakashima, K. Nagai, T. Tanaka, ${ }^{\text {a) }}$ and K. Matsuyama \\ Kyushu University, Department of Electronics, Motooka 744, Nishi-ku, Fukuoka 819-0395, Japan
}

(Presented 16 November 2010; received 24 September 2010; accepted 9 November 2010; published online 25 March 2011)

\begin{abstract}
In the present study, the operational modes and the structural design are optimized to realize potential performance in the interferometric spin wave logic circuits. Successive functional operations, such as generation, propagation, and inductive detection of spin wave packets are numerically studied by using micromagnetic simulations. The logic input is coded with the phase of pulsed microwave currents applied through the generators. Among the various kinds of the investigated spin wave (SW) modes, the backward volume mode exhibits superior performance. Various structural and operational parameters, including the pulsed microwave frequency and the film thickness of the magnetic strip, were optimized by taking the inductive output voltage $\left(V_{\text {out }}\right)$ as a quantitative criterion. The several orders of difference obtained in the $V_{\text {out }}$ for the different logic inputs demonstrates the successful exclusive-not-OR operation. (C) 2011 American Institute of Physics. [doi:10.1063/1.3549438]
\end{abstract}

\section{INTRODUCTION}

Magnetostatic spin waves (MSW) have recently attracted practical interest as novel information carriers in spintronics devices. Spin wave interferometers and current controlling of the phase were studied with Mach-Zehnder-type device structures. ${ }^{1-3}$ Another type of magnetic logic device utilizing the interference between the spin wave packets (SWPs) has also been proposed. ${ }^{4,5}$ Deep understanding of the dynamic properties of the SWP and optimum design consideration are key issues to realize the spin based interferometric devices. In the present study, fundamental device operations of nucleation, propagation, mutual interference and inductive detection of SWPs are numerically studied by using micromagnetic simulations. The device performances are also compared for the three different spin wave modes of magnetostatic surface wave (MSSW), magnetostatic forward volume wave (MSFVW), and magnetostatic backward volume wave (MSBVW).

\section{NUMERICAL SIMULATIONS}

Cooperative spin dynamics were investigated by solving the Landau-Lifshitz-Gilbert equation with a finite differential method. The accuracy of the numerical simulation was determined from comparison with the previously reported experimental results. ${ }^{6}$ The experimentally observed spin wave packet group velocity of $13.1 \mathrm{~km} / \mathrm{s}$ reasonably agrees with the simulation result of $12.8 \mathrm{~km} / \mathrm{s}$, assuming the same material parameters and device structures. In the following simulations standard material parameters for a ferromagnetic material are assumed; the saturation magnetization, exchange stiffness

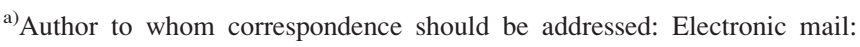
t-tanaka@ed.kyushu-u.ac.jp.
}

constant, and Gilbert damping constant are assumed to be 680 $\mathrm{emu} / \mathrm{cm}^{3}, 3.0 \times 10^{-7} \mathrm{erg} / \mathrm{cm}$ and 0.01 , respectively. The longitudinal bias field $H_{\mathrm{b}, \mathrm{x}}$, the transverse bias field $H_{\mathrm{b}, \mathrm{y}}$, and the perpendicular anisotropy field $H_{\mathrm{k}, \mathrm{z}}$ are assumed to define the precession axis for the MSBVW, MSSW and MSFVW modes, respectively. The values of $H_{\mathrm{b}, \mathrm{x}}, H_{\mathrm{b}, \mathrm{y}}$, and $H_{\mathrm{k}, \mathrm{z}}$ are chosen so that the ferromagnetic resonance frequency of the uniform mode $(k=0)$ becomes identical $(7.9 \mathrm{GHz})$ for the three configurations.

The schematic figure of designed device structure is shown in Fig. 1. A ferromagnetic strip with $82 \mu \mathrm{m}$ length and $5 \mu \mathrm{m}$ width was assumed as a spin wave guide. Hair-pin shape conductors (SWG1, SWG2) with $0.3 \mu \mathrm{m}$ width and $0.2 \mu \mathrm{m}$ gap are used for the generation of the SWPs. The SWPs are nucleated with nonuniform Oersted fields induced from the one cycle application of pulsed microwave current applied through the generators. The initial phase angle $\varphi$ of the pulsed microwave is used as the bit information. The data " 1 " and " 0 " are coded by $\varphi=0$ and $\pi$ rad. The interferometric operation is verified from the inductive output voltage $V_{\text {out }}$ calculated from the time differential of the whole magnetic flux $\Phi$ inside the spin wave detector with $0.2 \mu \mathrm{m}$ gap width, where the SWPs emitted from the two generators are superposed. The frequency of the pulsed microwave and the

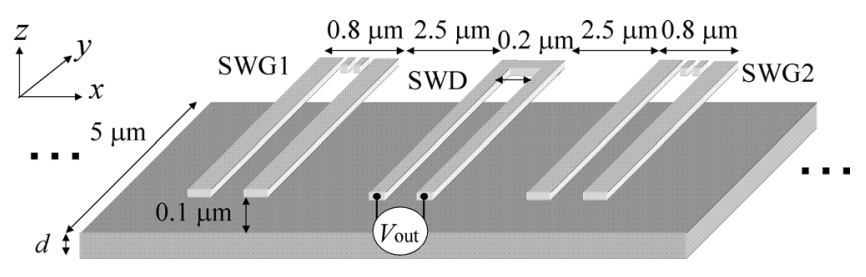

FIG. 1. Schematic figure of the interferometric spin wave logic device, constructed with the spin wave guide of a ferromagnetic strip, spin wave generators (SWG1, SWG2) and the spin wave detector (SWD). 

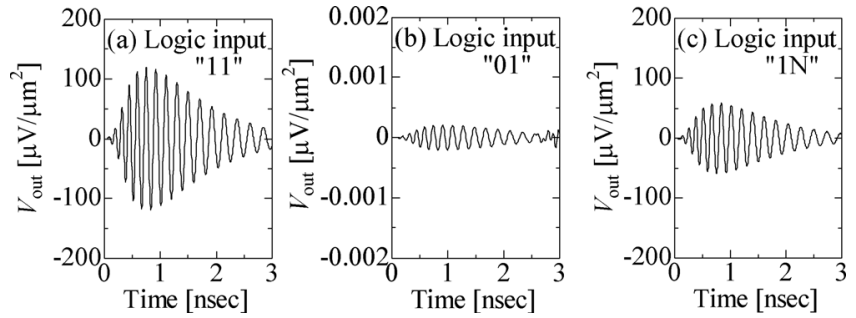

FIG. 2. Wave forms of inductively detected output voltage in MSBVW mode for different logic inputs; (a) "11", (b) " 01 ", and (c) " $1 \mathrm{~N}$ ". The logic data " 1 " and " 0 " are coded by the phase angle $\varphi=0$ and $\pi$ of the pulsed microwave current for the spin wave packet generation. The notation "N" means no input.

film thickness of the SW guide are optimized for three different spin wave modes so that the $V_{\text {out }}$ for a single SWP emitted with the same conductor current amplitude becomes maximum. The optimized values of $f_{\mathrm{g} \text {, opt }}$ and $d_{\mathrm{opt}}$ for the three spin wave modes are $8.9 \mathrm{GHz}$ and $100 \mathrm{~nm}$ for MSSW, $7.9 \mathrm{GHz}$ and $130 \mathrm{~nm}$ for MSFVW, and $6.9 \mathrm{GHz}$ and $900 \mathrm{~nm}$ for MSBVW, respectively.

\section{RESULTS AND DISCUSSION}

The SWP logic operations performed for the MSBVW mode are shown in Fig. 2, where the waveforms of the $V_{\text {out }}$ are plotted for various logic inputs. The notation " $\mathrm{N}$ " means no input. The $V_{\text {out }}$ for the logic input " 11 " is almost twice that for the single SWP ("1N"), which demonstrates the superposition of the two SWPs with the same $\varphi$. Several orders of smaller $V_{\text {out }}$ for the logic input "01" represents the cancellation of the two SWPs with the relative phase difference of $\pi$ rad. In contrast, the values of $V_{\text {out }}$ for the MSSW mode are markedly different depending on the propagation direction of SWP, as shown in Figs. 3(a) and 3(b). Resultantly, the cancellation for the out of phase SWP was imperfect, as shown in Fig. 3(c). The results originated from the nonreciprocal properties of the SWP emission in the MSSW mode. ${ }^{6}$

Figure 4 shows the deterioration of the logic performance due to the dislocation of the detector position. Since the relative phase angle at the detector depends on the propagation distance of the SWP, the maximum output voltage $V_{\text {out, } \max }$ for the logic input " 11 " decreases with the increase of the dislocation distance $t_{\mathrm{x}}$ and vice versa for " 01 ". Although the structural dislocation results in the degraded interferometric operation, the $t_{\mathrm{x}}$ of $0.1 \mu \mathrm{m}$ still exhibits a

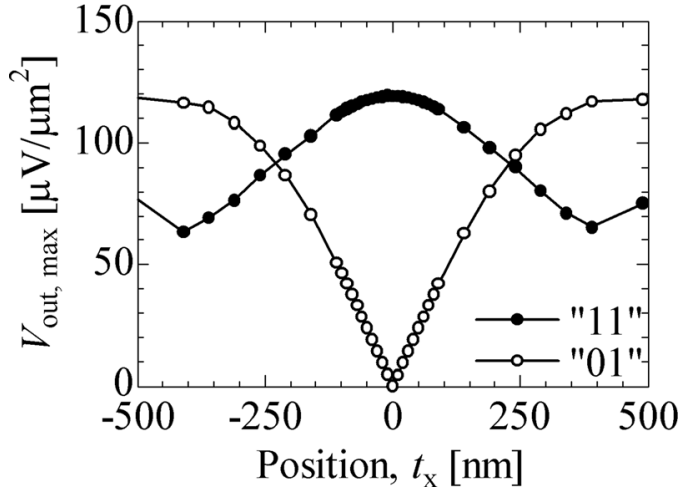

FIG. 4. Maximum output voltage as a function of the dislocation distance $t_{\mathrm{x}}$ of the detector. The dislocation affects the relative phase difference at the detector, which modulates the interferometric output.

practical $V_{\text {out, max }}$ difference of $113 \mu \mathrm{V} / \mu \mathrm{m}^{2}$ for the input " 11 " and $47 \mu \mathrm{V} / \mu \mathrm{m}^{2}$ for " 01 ".

The ratio of $V_{\text {out, } \max }$ for the input " 11 " and " 01 " is plotted in Fig. 5 as a function of $t_{\mathrm{x}}$. The results for the MSBVW and MSFVW modes are compared in the figure. Superior logic operation is realized in the MSBVW, where the ratio of $V_{\text {out, max }}$ for the two logic inputs reaches $115 \mathrm{~dB}$ at $t_{\mathrm{x}}=0 \mu \mathrm{m}$. This excellent performance is due to the almost perfect cancellation for the logic input " 01 ", where the $V_{\text {out, max }}$ is only $0.21 \mathrm{nV} / \mu \mathrm{m}^{2}$.

A soliton like behavior of the colliding SWPs is demonstrated in Fig. 6. In the simulation, the detector position is artificially dislocated at $t_{\mathrm{x}}=-2 \mu \mathrm{m}$ so that the SWPs from both sides of the generators can be individually detected with a time delay. Fig. 6(a) presents the reference $V_{\text {out }}$ for the single SWP. The first and second $V_{\text {out }}$ signals shown in Fig. 6(b) come from the SWPs generated at the left- and right- hand side generators. No distinguishable difference is observed for the $V_{\text {out }}$ in Figs. 6(a) and 6(b), noted as SWG2 in the figure. The results indicate that the collision of the SWPs does not affect the following propagation.

Figure 7 presents an interaction of the propagating SWP with the current induced pulsed magnetic field. An additional control conductor with the same design as the generator is located at the mid-to-mid $1 \mu \mathrm{m}$ separation from the generator. The simulations are performed for the pulsed microwave control currents with opposite polarities noted as $\varphi=0, \pi$ in the figure, and various delay times $T_{\mathrm{d}}$. As shown in the figure, the control fields enlarge or suppress the $V_{\text {out, max }}$, depending on the polarity and the values of $T_{\mathrm{d}}$. The
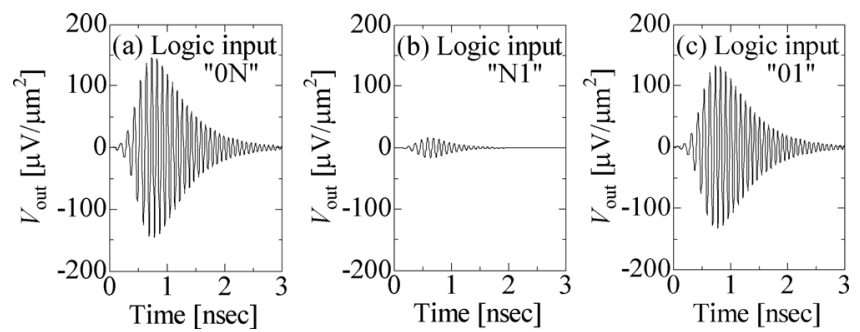

FIG. 3. Wave forms of inductively detected output voltage in MSSW mode for different logic inputs; (a) "0N", (b) "N1", and (c) "01".
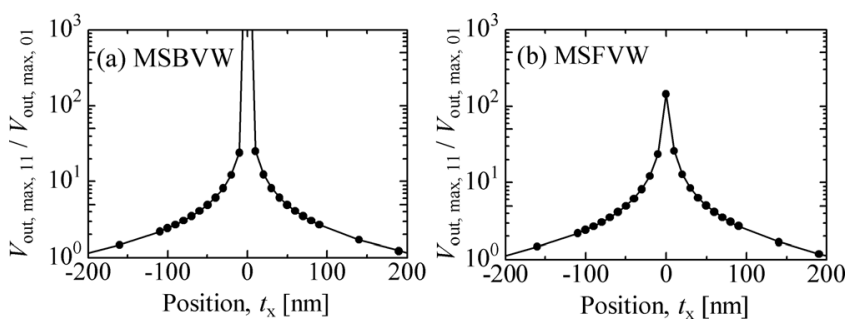

FIG. 5. Ratio of maximum output voltage for logic inputs of " 11 " and " 10 " versus the dislocation distance of detector; (a) MSBVW, (b) MSFVW. 

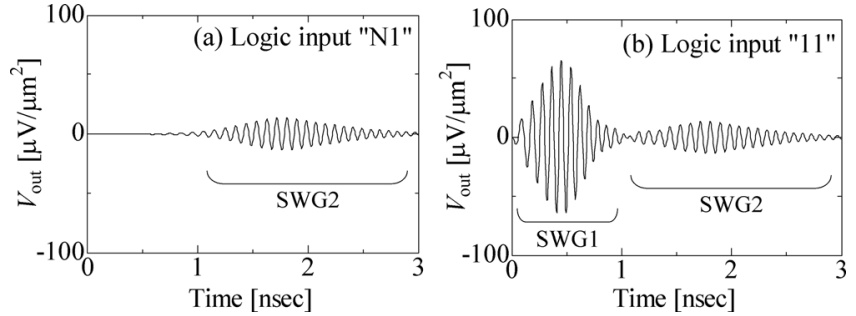

FIG. 6. Wave forms of inductively detected output voltage; (a) single spin wave packet, (b) two conflicting spin wave packets. Almost the same wave forms, noted as SWG2, suggest that the conflict causes no distinguishable influence on the spin wave packets propagation.

considerable modulation of the output signal amplitude with the control fields demonstrates the possibility of the amplification and the attenuation of the SWP in the logic circuit.

\section{CONCLUSION}

Interferometric logic operations utilizing spin wave packets were numerically studied. Fundamental operations of nucleation, propagation, interference, and inductive detection of spin wave packets are numerically studied by using micromagnetic simulations. The operation modes and the structural design were optimized to realize a practical performance. The logic inputs were coded by the relative phase difference of the pulsed microwave currents applied through the generators. Among the various investigated spin wave

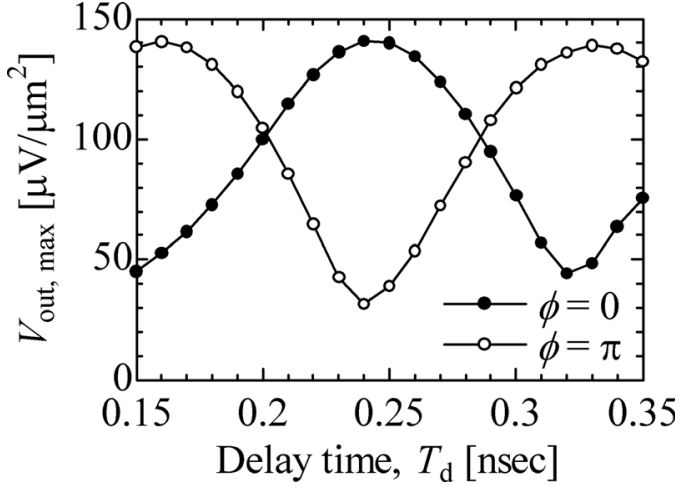

FIG. 7. Control the propagating spin wave packets with pulsed microwave current with opposite polarity $(\varphi=0, \pi)$ and various delay times $T_{\mathrm{d}}$. The current induced magnetic fields enhance or attenuate the precession of the spin wave, depending on the relative phase difference.

modes, the backward volume mode exhibited superior performance. The numerically predicted $115 \mathrm{~dB}$ difference in the output voltage for the logic inputs of " 11 " and " 01 " demonstrates the successful logic operation.

${ }^{1}$ Y. K. Fetisov and C. E. Patton, IEEE Trans. Magn. 35, 1024 (1999).

${ }^{2}$ M. P. Kostylev, A. A. Serga, T. Schneider, B. Leven, and B. Hillebrands, Appl. Phys. Lett. 87, 13501 (2005).

${ }^{3}$ T. Schneider, A. A. Serga, B. Leven, B. Hillebrands, R. L. Stamps, and M. P. Kostylev, Appl. Phys. Lett. 92, 022505 (2008).

${ }^{4}$ A. Khitun and K. L. Wang, Superlattices Microstruct. 38, 184 (2005).

${ }^{5}$ A. Khitun, M. Bao, and K. L. Wang, IEEE Trans. Magn. 44, 2141 (2008).

${ }^{6}$ K. Sekiguchi, K. Yamada, S. M. Seo, K. J. Lee, D. Chiba, K. Kobayashi, and T. Ono, Appl. Phys. Lett. 97, 022508 (2010). 